\section{Avaliação da testagem anti-HIV no pré-natal e na assistência ao parto no Rio de Janeiro, Brasil}

\section{An evaluation of HIV testing during pre- and perinatal care in Rio de Janeiro, Brazil}

\begin{abstract}
Introduction: to evaluate HIV testing during preand perinatal care in the Brazilian National Health System.

Methods: a cross-sectional study was carried out in 2009 covering 15 maternity hospitals in Rio de Janeiro. Interviews were conducted with a sample of 835 pregnant women and their medical records consulted. A logical model was drawn up to assess the adequacy of HIV testing.

Results: according to the information gathered from the pregnant women, $86.7 \%$ underwent a prenatal non-reactive serology test and $55.7 \%$ a rapid HIV test in hospital.In $49.9 \%$ of cases, the rapid hospital HIV test procedure was deemed to be adequate both for mothers with unknown prenatal $H I V$ status undergoing the rapid test and for those with known HIV status who did not undergo this test. According to medical records, $68.0 \%$ underwent the non-reactive serum testand $79.6 \%$ the rapid HIV test.In 50.9\%of cases the rapid hospital HIV test procedure was found to be adequate.

Conclusions: the pre- and perinatal HIV test protocol in force in 2009 was not followed in a satisfactory manner, as unnecessary tests were performed and the target population was not fully tested, thereby jeopardizing the adoption of adequate prophylactic measures to control vertical transmission.
\end{abstract}

Key words Program evaluation, HIV, Prenatal care, AIDS serodiagnosis, Cross-sectional studies, Unified Health System
Daniela Marcondes Gomes 1

Maria Inês Couto de Oliveira 2

Sandra Costa Fonseca 3

${ }^{1}$ Programa de Pós-Graduação em Saúde Coletiva. Instituto de Saúde Coletiva. Universidade Federal Fluminense. Rua Marques de Paraná, $\mathrm{n}^{\circ} 303$ anexo. $4^{\circ}$ andar. Centro. Niterói, RJ, Brasil. CEP: 24.033-900. E-mail: dn.marcondes@ig.com.br

2,3 Departamento de Epidemiologia e Bioestatística. Instituto de Saúde Coletiva. Universidade Federal Fluminense. Niterói, RJ, Brasil.

\section{Resumo}

Objetivos: avaliar a testagem anti-HIV durante a assistência pré-natal e ao parto no Sistema Único de Saúde.

Métodos: estudo transversal conduzido em 2009 em 15 maternidades no Rio de Janeiro, sendo entrevistada amostra representativa de 835 parturientes e observados prontuários. Para avaliação da adequação da testagem anti-HIV foi elaborado um modelo lógico.

Resultados: segundo informação das parturientes, $86,7 \%$ dispunham de sorologia não reagente do pré-natal e 55,7\% foram submetidas ao teste rápido anti-HIV no hospital; em 49,9\% dos casos o procedimento relativo ao teste rápido anti-HIV no hospital foi considerado adequado: mães com status ignorado de HIV do pré-natal submetidas ao teste rápido e mães com status conhecido não submetidas ao mesmo. Segundo dados do prontuário, 68,0\% dispunham de sorologia não reagente e 79,6\% foram submetidas ao teste rápido anti-HIV; em 50,9\% dos casos o procedimento relativo ao teste rápido antiHIV no hospital foi adequado.

Conclusões: o protocolo de exames anti-HIV no pré-natal e na maternidade, vigentes em 2009, não foram cumpridos a contento, tanto por gerar procedimentos desnecessários quanto falhas na testagem da população alvo, ameaçando a instituição oportuna de medidas profiláticas de controle da transmissão vertical.

Palavras-chave Avaliação de programas e projetos de saúde, HIV, Assistência pré-natal, Sorodiagnóstico da AIDS, Estudos transversais, Sistema Único de Saúde 


\section{Introdução}

Na década de 1990 foi iniciada a organização das ações de prevenção da transmissão vertical da infecção pelo HIV no mundo, com foco nas gestantes de risco. Posteriormente, estas ações assumiram caráter universal, levando à possibilidade de controle e eliminação da AIDS infantil. ${ }^{1}$

A transmissão vertical do HIV pode ocorrer durante a gestação e parto e/ou pela amamentação, podendo as taxas de transmissão variar entre $25 \%$ e $30 \%$, caso as medidas preventivas preconizadas não sejam oportunamente instituídas.1,2 $\mathrm{O}$ estabelecimento de tais medidas determina uma redução dos percentuais de transmissão a níveis inferiores a $2 \% .{ }^{2}$

Barreto et al. 3 avaliaram sucessos e fracassos no controle da epidemia HIV/AIDS no Brasil e concluíram que as iniciativas de controle da transmissão vertical do HIV, como a oferta de testes diagnósticos gratuitos e a profilaxia nos serviços de prénatal, foram parcialmente exitosas. Quanto às falhas, os autores consideraram que vêm sendo compensadas pela realização, em escala nacional, de testes rápidos de detecção da infecção pelo HIV em parturientes não testadas durante o pré-natal e pelo aumento substancial da sobrevida e melhora da qualidade de vida das crianças vivendo com HIV/AIDS. 3

No Brasil, a vigilância da transmissão vertical do vírus HIV é feita a partir do monitoramento dos coeficientes de detecção de casos de AIDS em menores de cinco anos. ${ }^{4}$ Esta taxa, que era de $4,2 / 100.000$ em 2004, passou a 2,7/100.000 em 2013, o que representa uma redução de $35,7 \%$ em uma década. ${ }^{4}$ Entretanto, estudos populacionais têm mostrado desigualdades na redução da transmissão vertical no país. No Estado de São Paulo, os coeficientes de detecção passaram de $9,4 \%$, em 2000 , para 2,7\%, em 2006,5 correspondendo a uma queda de $70 \%$, enquanto que região da Baixada Fluminense, no Estado do Rio de Janeiro, observouse uma taxa média de 4,7\% de transmissão vertical, ${ }^{6}$ sem tendência de queda, no período de 1999-2009.

Ainda no nível nacional, a prevalência de infecção por HIV em gestantes foi estimada em $0,40 \%$ entre 2011 e 2012.7 A taxa de detecção da infecção pelo vírus HIV tem sofrido aumento, passando de 2,0 casos/1.000 nascidos vivos (mil $\mathrm{NV}$ ) em 2004, para 2,5 casos/mil NV em 2013. Esta tendência crescente vem sendo observada em todas as regiões brasileiras, exceto no Sudeste. ${ }^{4}$ O Estado do Rio de Janeiro apresenta a quarta maior taxa de detecção de gestantes com HIV, de 3,0 casos/ mil NV. 4
Em 2014, a Organização Pan-Americana da Saúde (OPAS) criou o Comitê Regional para Validação da Eliminação da Transmissão MaternoInfantil de HIV, 2 a ser conferido aos países que atingirem as metas de no mínimo de $95 \%$ de cobertura de testagem para a infecção pelo HIV de gestantes e de menos de $2 \%$ de taxas de transmissão vertical.

As rotinas de rastreamento de infecção pelo vírus HIV, durante a assistência pré-natal e ao parto no Brasil, estabelecidas pelo Ministério da Saúde (MS), estão descritas em vários documentos. O Manual Técnico de Pré-natal e Puerpério $(2006)^{8}$ e as Recomendações para Profilaxia da Transmissão Vertical do HIV (2007)9 ${ }^{9}$ recomendam a realização obrigatória de testes para detecção de anticorpos anti-HIV nas gestantes durante a primeira consulta de pré-natal, e sua repetição no início do $3^{\circ}$ trimestre, sempre que possível; o Protocolo para a Prevenção da Transmissão Vertical de HIV e Sífilis, publicado em 2007,10 preconiza a realização do teste rápido anti-HIV em todas as mulheres admitidas para o parto na maternidade, que não foram testadas durante pré-natal ou sem resultado disponível.

A partir de 2010, o MS passou a recomendar que gestantes não testadas no último trimestre de gestação passassem a ser submetidas ao teste rápido anti-HIV, além daquelas em situação de risco acrescido: soronegativas com parceiro infectado; que trocaram de parceiro ou que apresentaram Doenças Sexualmente Transmissíveis (DST) durante a gestação; profissionais do sexo; usuárias de álcool e outras drogas, independentemente do tempo decorrido desde o último teste anti-HIV. 11

Igualmente, o MS propôs a ampliação dos critérios de uso do teste rápido no pré-natal, em situações em que o diagnóstico da infecção pelo HIV não pode ser realizado em tempo hábil para a adoção de medidas de redução da transmissão vertical do vírus, como em regiões sem infraestrutura laboratorial ou de difícil acesso, ou nas gestantes cuja idade gestacional não assegure o recebimento do resultado do teste antes do parto. 11

Os testes rápidos para detecção de anticorpos anti-HIV detectam anticorpos contra o HIV em menos de 30 minutos, têm baixo custo, alta sensibilidade e especificidade, além de fácil realização e interpretação, 12 e foram incorporados à rotina diagnóstica nas maternidades, quando ainda é possível evitar a transmissão vertical. ${ }^{11}$

No município do Rio de Janeiro, medidas de prevenção da infecção pelo HIV foram iniciadas em 1996. No entanto, ainda se observam oportunidades perdidas de prevenção, 13 bem como o uso excessivo de testes rápidos no momento da admissão para o 
parto. ${ }^{14} \mathrm{O}$ objetivo deste estudo foi avaliar a adequação da testagem anti-HIV durante a assistência pré-natal e a internação para o parto em maternidades vinculadas ao Sistema Único de Saúde (SUS) no município do Rio de Janeiro, no ano de 2009.

\section{Métodos}

Trata-se de um estudo transversal desenvolvido para avaliar a adequação 15 das ações de prevenção da transmissão vertical do HIV, segundo os protocolos recomendados pelo MS. O estudo foi realizado com base nos dados de uma pesquisa cujo objetivo principal foi avaliar a implementação da Iniciativa Hospital Amigo da Criança no Município do Rio de Janeiro. 16

A população do estudo foi composta por uma amostra representativa de 835 parturientes internadas no segundo semestre de 2009, em hospitais do SUS que realizavam mais de 1000 partos por ano no município do Rio de Janeiro, um total de quinze. Destes quinze hospitais, sete eram credenciados na Iniciativa Hospital Amigo da Criança (HAC) e oito não credenciados (HNC). Onze destes hospitais (cinco HAC e seis HNC) faziam parte do Sistema de Gestação de Alto Risco, de referência materna e/ou fetal. Em 2008, esses serviços concentraram $94 \%$ do total de partos realizados em unidades hospitalares do SUS no município.

Empregou-se uma amostragem estratificada, cujos estratos eram os hospitais. Para o cálculo do tamanho amostral, optou-se por considerar uma prevalência arbitrária de $50 \%$ do desfecho principal da pesquisa, que foi o cumprimento de cada passo da Iniciativa Hospital Amigo da Criança. Uma prevalência de $50 \%$ foi utilizada como fator de ponderação para cada estrato, por garantir o maior tamanho de amostra possível para nível de erro e confiança controlados. 17 Para um nível de erro de $5 \%$ e de confiança de $99 \%$, obteve-se um tamanho mínimo de 687 mães em alojamento conjunto, distribuídas entre os estratos segundo a quantidade de partos em 2008. 16

Foram incluídas as mães com filhos nascidos vivos no hospital há mais de 24 horas. Como a amamentação era um dos principais desfechos da pesquisa, foram excluídas as mulheres com alguma contra-indicação à amamentação, como sorologia reagente para o HIV (previamente conhecida ou diagnosticada na maternidade). As entrevistadas foram selecionadas por sorteio sistemático a partir da ficha do posto de enfermagem. Parturientes sorteadas, cujos bebês estavam internados na unidade neonatal há mais de 48 horas foram igualmente incluídas no estudo, tendo sido incorporados à amostra um total de 148 mães de bebês internados. 16

Os dados foram obtidos entre agosto e dezembro de 2009 por meio de entrevista com as parturientes nas enfermarias de alojamento conjunto e extração de informações dos prontuários maternos. Os questionários estruturados continham perguntas sobre características da mãe, do domicílio, assistência pré-natal, ao parto, e sobre o recém-nato. O formulário de coleta de dados do prontuário foi utilizado para a obtenção de informações relativas ao parto e sobre a realização de testes para detecção da infecção pelo HIV durante o acompanhamento pré-natal e durante a internação para o parto. Os entrevistadores eram profissionais de saúde previamente treinados para a aplicação do questionário.

Para avaliação da adequação das ações de prevenção da transmissão vertical do HIV, foi elaborado um modelo lógico18 do rastreamento da infecção pelo HIV durante a assistência pré-natal e ao parto, utilizando como referência os protocolos ministeriais vigentes no período de coleta de dados (2009). 9,10 Este modelo compreendeu o contexto organizacional, as situações problema relativas à inadequação das práticas assistenciais de controle do HIV, a estrutura necessária para este controle, as atividades pertinentes e sua população alvo, bem como os desfechos a curto, médio e longo prazo (Figura 1).

As seguintes informações foram obtidas por entrevista com as mães: época do início do pré-natal, número de consultas pré-natais, resultado de exame anti-HIV do pré-natal e submissão ao teste rápido anti-HIV no hospital (Figura 2). Por observação do prontuário foram colhidos dados sobre o resultado de exame anti-HIV do pré-natal e a submissão ao teste rápido anti-HIV no hospital (Figura 3).

As seguintes ações foram consideradas adequadas: realização de teste rápido nas mães com status ignorado de infecção por HIV (não fizeram pré-natal, não fizeram exame anti-HIV no pré-natal, ou não receberam/não sabiam o resultado do exame anti-HIV do pré-natal) e a não submissão de mães com status conhecido de HIV do pré-natal (Figuras 2 e 3).

Não foi calculada a proporção de mulheres com exame realizado no $1^{\circ}$ trimestre (ou início do prénatal) e no $3^{\circ}$ trimestre, pois em $38,9 \%$ dos prontuários constava o resultado negativo do prénatal, mas não a data deste exame. A adequação da 
Modelo lógico da testagem anti-HIV durante a assistência pré-natal e ao parto.

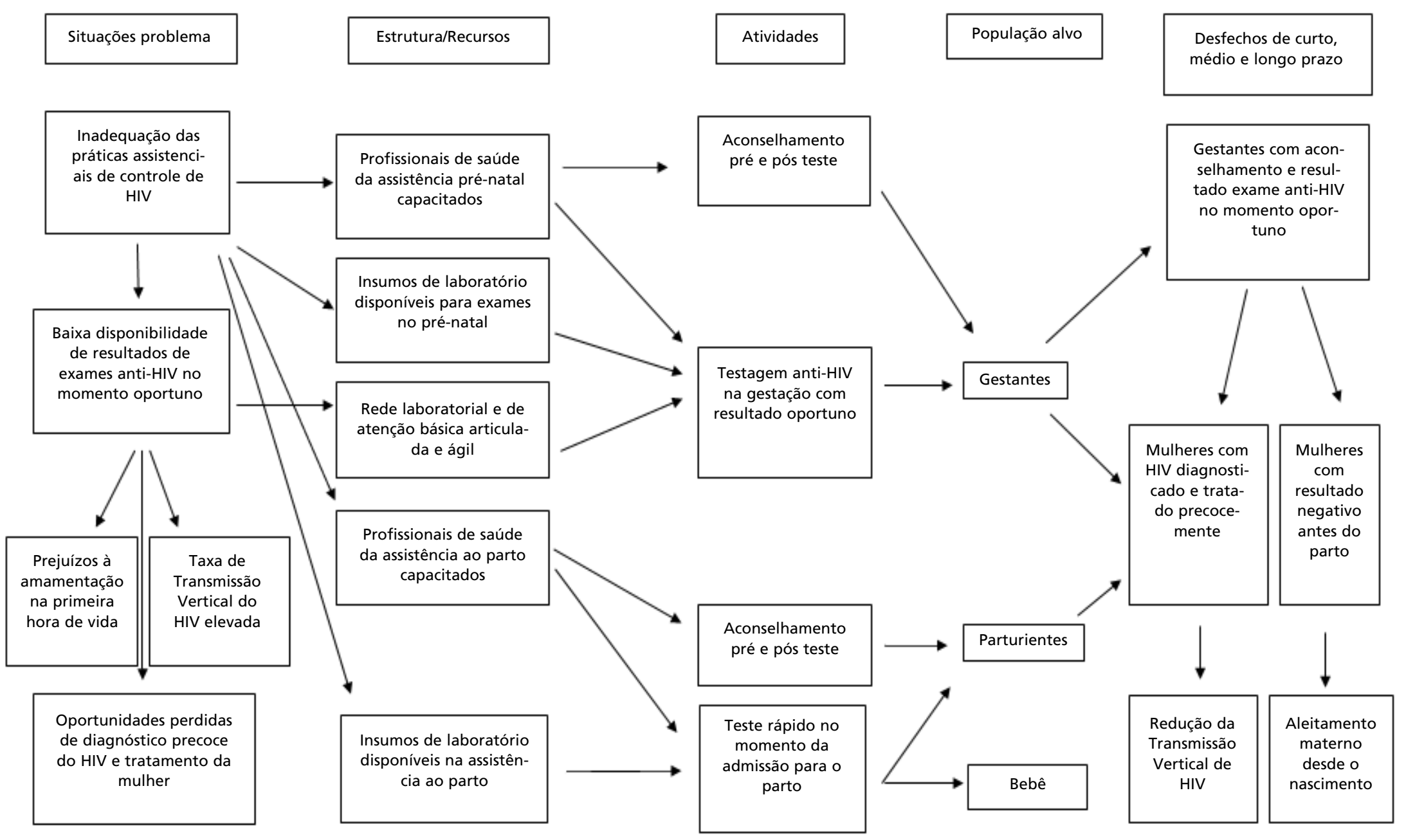




\section{Figura 2}

Modelo de avaliação da adequação das ações de controle do HIV durante a assistência pré-natal e ao parto, segundo a informação das parturientes internadas em hospitais do Sistema Único de Saúde. Município do Rio de Janeiro, 2009.
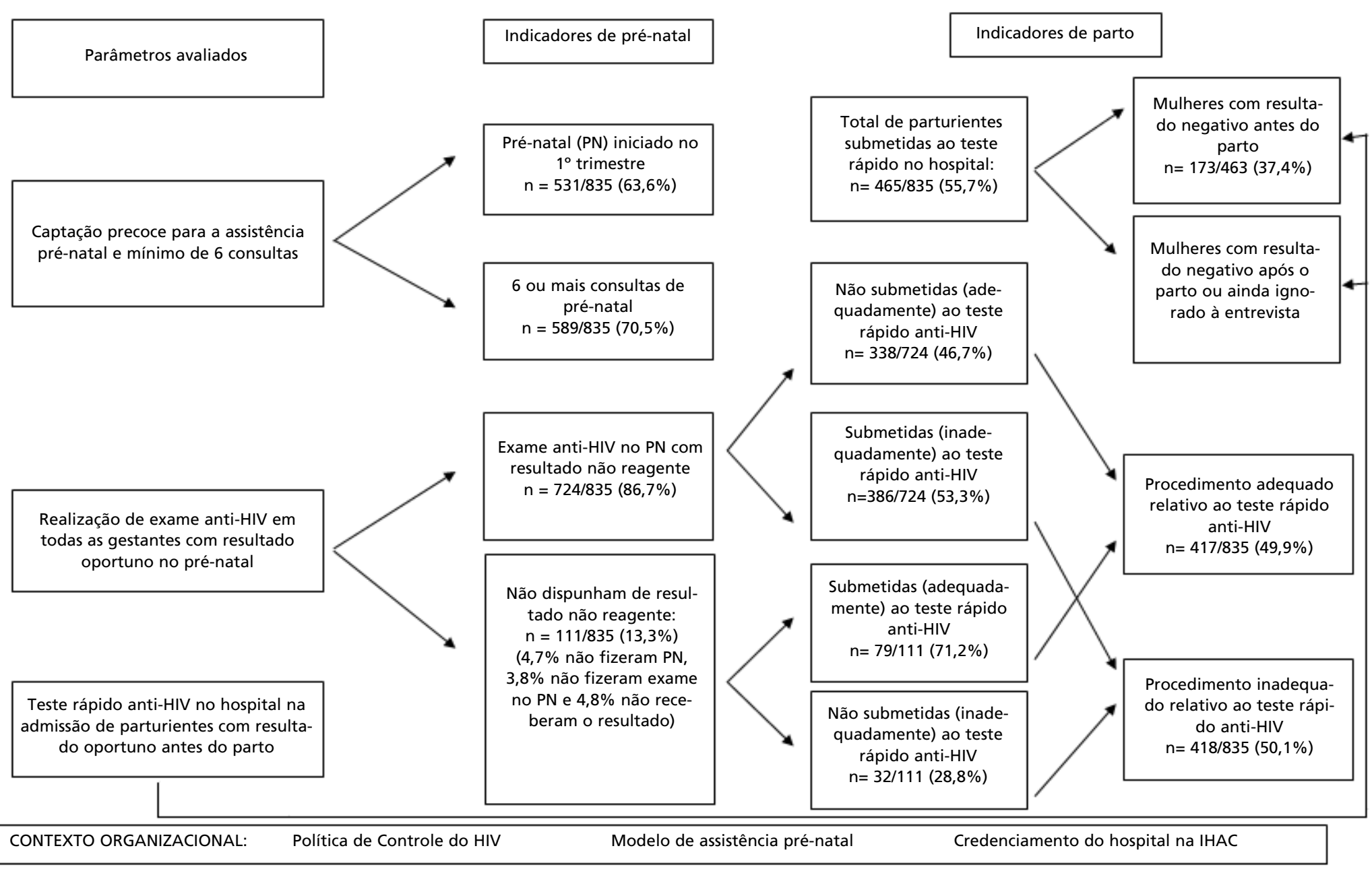
Modelo de avaliação da adequação das ações de controle do HIV durante a assistência pré-natal e ao parto, segundo informação do prontuário das parturientes internadas em hospitais do Sistema Único de Saúde. Município do Rio de Janeiro, 2009.

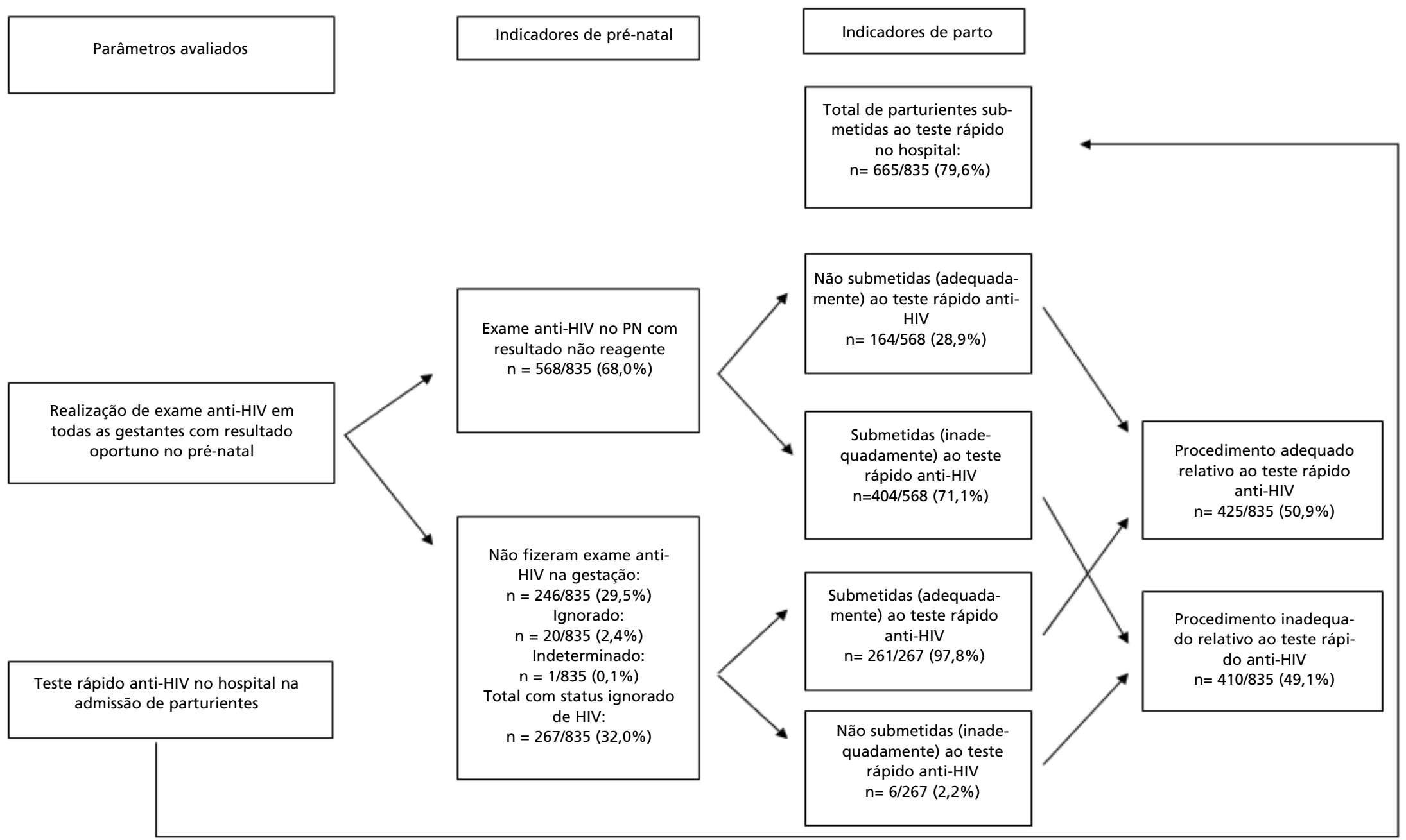


submissão ao teste rápido anti-HIV foi calculada com os dados das duas fontes de informação: entrevistas e prontuários.

O cálculo das frequências e proporções foi realizado utilizando o programa SPSS, versão 17 (Statistical Package for Social Sciences).

A pesquisa foi avaliada e aprovada pelo Comitê de Ética e Pesquisa da Secretaria Municipal de Saúde do Rio de Janeiro (parecer nº 77A/2009).

\section{Resultados}

Foram excluídas três mães com sorologia positiva para o HIV. Houve sete recusas e nove perdas (puérperas que já tinham recebido alta no momento da entrevista) que foram repostas pela mãe subsequente no sorteio. $\mathrm{Na}$ amostra final, foram entrevistadas 687 mães em alojamento conjunto e 148 mães com bebês internados em unidade neonatal.

Mais de um quarto das mães era adolescente, e um terço não havia completado o ensino fundamental; a metade tinha trabalho remunerado e dois terços apresentaram renda de até um salário mínimo; a maioria tinha companheiro, quase a metade era primípara, e um quarto residia em domicílios com seis ou mais moradores (Tabela 1).

A maior parte das mães tinha realizado pré-natal $(95,3 \%)$, das quais cerca de dois terços em unidade básica de saúde. Quanto à assistência ao parto, $63,5 \%$ tiveram seus filhos de parto normal e a metade dos partos ocorreu em hospitais certificados na Iniciativa Hospital Amigo da Criança (Tabela 2).

\section{Tabela 1}

Características sociodemográficas das parturientes internadas em hospitais do Sistema Único de Saúde. Município do Rio de Janeiro, 2009.

\begin{tabular}{|c|c|c|}
\hline Variáveis & $\mathbf{N}$ & $\%$ \\
\hline \multicolumn{3}{|l|}{ Idade (anos) } \\
\hline 20 a 46 & 618 & 74,0 \\
\hline 13 a 19 & 217 & 26,0 \\
\hline \multicolumn{3}{|l|}{ Cor da pele } \\
\hline Branca & 219 & 26,2 \\
\hline Não branca & 616 & 73,8 \\
\hline \multicolumn{3}{|l|}{ Escolaridade } \\
\hline$\geq$ Ensino fundamental completo & 555 & 66,5 \\
\hline Ensino fundamental incompleto & 280 & 33,5 \\
\hline \multicolumn{3}{|l|}{ Trabalho remunerado } \\
\hline Sim & 422 & 50,5 \\
\hline Não & 413 & 49,5 \\
\hline \multicolumn{3}{|l|}{ Renda materna } \\
\hline > 1 salário mínimo & 278 & 33,3 \\
\hline$\leq 1$ salário mínimo & 555 & 66,7 \\
\hline \multicolumn{3}{|l|}{ Companheiro } \\
\hline Tem companheiro & 723 & 86,6 \\
\hline Não tem companheiro & 112 & 13,4 \\
\hline \multicolumn{3}{|l|}{ Paridade } \\
\hline Primípara & 399 & 47,8 \\
\hline Não primípara & 436 & 52,2 \\
\hline \multicolumn{3}{|l|}{ Número de moradores } \\
\hline Até 5 & 625 & 74,9 \\
\hline 6 ou mais & 210 & 25,1 \\
\hline
\end{tabular}


Tabela 2

Características da assistência pré-natal e ao parto de parturientes internadas em hospitais do Sistema Único de Saúde. Município do Rio de Janeiro, 2009.

\begin{tabular}{|c|c|c|}
\hline Variáveis & $\mathbf{N}$ & $\%$ \\
\hline \multicolumn{3}{|l|}{ Fez pré-natal } \\
\hline Sim & 796 & 95,3 \\
\hline Não & 39 & 4,7 \\
\hline \multicolumn{3}{|l|}{ Onde fez pré-natal } \\
\hline Em unidade básica & 523 & 65,7 \\
\hline Em outros serviços & 273 & 34,3 \\
\hline \multicolumn{3}{|l|}{ Início do pré-natal } \\
\hline $1^{\circ}$ trimestre & 531 & 63,6 \\
\hline $2^{\circ}$ ou $3^{\circ}$ trimestre ou não fez pré-natal & 304 & 36,4 \\
\hline \multicolumn{3}{|l|}{ Número de consultas de pré-natal } \\
\hline 6 ou mais consultas & 589 & 70,6 \\
\hline 0 a 5 consultas & 245 & 29,4 \\
\hline \multicolumn{3}{|l|}{ Realização de exame anti-HIV na gestação } \\
\hline $\operatorname{Sim}$ & 764 & 91,5 \\
\hline Não ou não sabe ou não fez pré-natal & 71 & 8,5 \\
\hline \multicolumn{3}{|l|}{ Tipo de parto } \\
\hline Normal & 530 & 63,5 \\
\hline Cesariana & 305 & 36,5 \\
\hline \multicolumn{3}{|l|}{ Parto em Hospital Amigo da Criança } \\
\hline $\operatorname{sim}$ & 424 & 50,8 \\
\hline Não & 411 & 49,2 \\
\hline
\end{tabular}

$\mathrm{Na}$ avaliação de adequação verificou-se que menos de dois terços das mães iniciaram o pré-natal no primeiro trimestre de gestação e quase $30 \%$ foram assistidas por menos de seis consultas. Segundo a informação materna, $86,7 \%$ das parturientes dispunham de resultado não reagente da gestação e $55,7 \%$ foram submetidas ao teste rápido anti-HIV no hospital (Figura 2). Segundo dados do prontuário, $68,0 \%$ dispunham de resultado não reagente do exame anti-HIV da gestação e $79,6 \%$ foram submetidas ao teste rápido anti-HIV (Figura 3).

Segundo os dados das entrevistas, em $49,9 \%$ dos casos o procedimento relativo ao teste rápido antiHIV no hospital foi considerado adequado: mães com status ignorado de HIV do pré-natal foram submetidas ao teste rápido, enquanto mães com status conhecido não fizeram o teste. (Figura 2). Pelos dados do prontuário, em 50,9\% dos casos o procedimento relativo ao teste rápido anti-HIV no hospital foi adequado, de acordo com os mesmos critérios (Figura 3).

Ainda segundo a informação materna, apenas $37,4 \%$ das mães submetidas ao teste rápido anti-HIV no hospital receberam seu resultado antes do parto (Figura 2).

\section{Discussão}

Apesar de a cobertura pré-natal ter sido alta, cerca de um terço das gestantes não foi captado precocemente para a assistência pré-natal e não foi acompanhado pelo número mínimo de consultas prénatais preconizados pelo Ministério da Saúde. ${ }^{8}$

$\mathrm{O}$ aumento da testagem da infecção pelo vírus do HIV durante o pré-natal é uma das estratégias para se alcançar o nível de cuidado "ideal" para a redução da transmissão vertical, aliado ao aumento do alcance do tratamento e da adesão à terapia antirretroviral. Em um estudo de simulação das condições para atingir a meta de redução da transmissão vertical da OPAS, 2 o percentual de testagem durante o pré-natal teria que atingir ainda mais que 95\% em algumas regiões do Brasil. 19 No entanto, segundo as informações maternas, $13,3 \%$ das parturientes não fizeram ou não dispunham de resultado negativo de qualquer exame anti-HIV, e 
segundo as informações do prontuário, este percentual atingiu $32,0 \%$. Esta disparidade pode se dever a mulheres que não apresentaram o cartão de pré-natal na admissão à maternidade, e com isto o sorodiagnóstico relatado da gestação não pode ser considerado pela equipe hospitalar. Porém, pelo menos $13 \%$ das mulheres não dispunham de sorologia anti-HIV da gravidez.

Oportunidades perdidas de diagnóstico precoce na gestação têm sido uma constante, identificadas em investigações nacionais $20-23$ e regionais. ${ }^{13,24-27}$ No Rio de Janeiro, dados de 2000 mostraram que $25 \%$ das gestantes não realizaram exame anti-HIV no pré-natal, 24 enquanto dados colhidos entre $2007 \mathrm{e}$ 2008 revelaram ausência do resultado deste exame em $26 \%$ dos cartões de pré-natal das gestantes com mais de 37 semanas gestacionais. 13 Se utilizarmos a informação dos prontuários, houve certa piora do indicador, mas se nos basearmos na informação materna, reduziu-se o percentual de mulheres sem o exame.

A OPAS recomenda uma cobertura mínima de $95 \%$ de mulheres testadas para o HIV na gravidez. ${ }^{2}$ Explicações para a cobertura não atingida de gestantes com sorologia anti-HIV podem estar relacionadas a barreiras ainda existentes para a realização e para o início precoce do pré-natal e à morosidade do retorno dos resultados em tempo oportuno. Estudos brasileiros apontam uma ampla variação da cobertura de testagem anti-HIV na gestação, de 8,4\% em Feira de Santana/BA26 a 99,5\% em Rio Grande/RS.28

Também ocorreram perda de oportunidades na assistência hospitalar, embora com grande discordância entre as fontes dos dados. Das 111 mulheres sem qualquer resultado de exame anti-HIV da gestação, $32(28,8 \%)$ não foram submetidas ao teste rápido anti-HIV, segundo informações maternas. No entanto, de acordo com os dados do prontuário, ocorreram apenas $2,2 \%$ de oportunidades perdidas de submissão ao teste rápido anti-HIV, representando um avanço em relação a estudos anteriores, pois para esta informação no âmbito hospitalar, o registro em prontuário é considerado como o padrão ouro. Em Feira de Santana entre 2003 e $2004,2682,3 \%$ das gestantes não testadas no prénatal deixaram de ser submetidas ao teste rápido anti-HIV, em Recife, na mesma época, este percentual foi de $15,5 \%{ }^{29}$ e no estudo "SentinelaParturiente", de âmbito nacional, 18\% das mulheres não haviam sido testadas para o HIV durante a gestação e na maternidade. 22

A discordância entre as informações do prontuário e das mães sugere que este avanço na redução das oportunidades perdidas de testagem rápida não apresentou correspondência com a interpelação das mulheres pela equipe de saúde. Segundo os protocolos do MS, as mulheres devem ser consultadas, mediante aconselhamento, se querem ser submetidas ao teste anti-HIV. ${ }^{9}$ Provavelmente, muitas parturientes submetidas ao teste rápido anti-HIV não foram sequer comunicadas desta submissão, e os resultados correspondentes não chegaram a seu conhecimento em tempo oportuno, sugerindo uma menor autonomia da mulher.

Em 2009, o número de mulheres submetidas ao teste rápido anti-HIV na admissão para o parto foi elevado. Estudo nacional e investigação conduzida no mesmo município, ambos em 2006, encontraram, respectivamente, proporções de $18,9 \% 22$ e $28,5 \% 14$ de parturientes submetidas ao teste rápido anti-HIV na internação para o parto, sinalizando que a submissão de mulheres a este teste vem ocorrendo em escala crescente no nosso país.

Considerando as supracitadas normas ministeriais, 9,10 foi alta a proporção de mulheres submetidas desnecessariamente ao teste rápido anti-HIV no hospital, por disporem de sorologia negativa do prénatal. Esta proporção foi de $53,3 \%$, segundo as entrevistas, e de $71,1 \%$, segundo dados dos prontuários. Os testes rápidos implicam em gastos com material de consumo e tempo dos profissionais de saúde. A realização rotineira do teste rápido antiHIV nas maternidades contribui para o retardo de seus resultados, prejudicando as ações de prevenção da transmissão vertical e postergando o aleitamento materno para além da primeira hora de vida, 14 haja vista que no presente estudo apenas $37,4 \%$ das mulheres submetidas ao teste rápido relataram ter recebido seu resultado antes do parto. Por outro lado, a ampliação do uso do teste rápido anti-HIV no prénatal, especialmente em mulheres no terceiro trimestre de gestação sem exame anterior, seria uma medida importante para a redução da transmissão vertical do HIV. ${ }^{11}$

Devemos considerar algumas limitações do presente estudo. A discrepância entre os dados obtidos das duas fontes de informação - entrevista e consulta a prontuário - sinaliza a possibilidade de viés de informação quanto à realização do exame anti-HIV na gestação e à submissão ao teste rápido anti-HIV na internação para o parto. Segundo informações do prontuário o percentual de mulheres testadas quanto ao HIV no pré-natal foi $18,7 \%$ inferior e o percentual de mulheres submetidas ao teste rápido anti-HIV foi $25 \%$ superior ao obtido pela informação materna. Discordâncias quanto à existência de resultado do primeiro exame anti-HIV 
segundo a entrevista com gestantes $(80,2 \%)$ e registro no cartão de pré-natal $(59,2 \%)$ foram observadas, da mesma forma que em outro estudo realizado no município do Rio de Janeiro ${ }^{13}$ e decorrem tanto da falta de comunicação entre a equipe e a clientela, quanto ao registro incompleto de informações no prontuário. Esta limitação de informação também não permitiu que fossem comparados os dados de testagem anti-HIV por trimestre gestacional segundo as entrevistas e o prontuário.

As mulheres soropositivas para o HIV não foram objeto do presente estudo, apesar da sua importância para a avaliação das ações de controle da transmissão vertical do HIV. No entanto, no Brasil a prevalência de HIV entre gestantes é baixa4 e vários estudos têm focado esta população, 7,25,30 enquanto poucos têm se preocupado em investigar a adequação da testagem anti-HIV.14,26

Convém destacar que gestantes em situação de risco acrescido ou vulnerabilidade, mesmo tendo sido testadas no início da gestação para o HIV, devem ser testadas novamente no final da gestação ou na internação para o parto.8,11 Estas circunstâncias não foram resgatadas no presente estudo por dificuldade de acesso à informação se as mulheres se enquadravam nestes critérios, podendo assim ter ocorrido uma subestimação das indicações de emprego do teste rápido anti-HIV. No entanto, podese presumir que mulheres portadoras destas condições, não investigadas adequadamente no prénatal, seriam em pequeno número, e, portanto, a magnitude desta subestimação não afetaria de forma relevante o cálculo do percentual de testes realizados desnecessariamente.

Conclui-se que há falhas na prevenção da transmissão vertical do HIV na cidade do Rio de Janeiro. Em primeiro lugar, destaca-se o não cumprimento dos protocolos ministeriais pelos profissionais de saúde envolvidos no cuidado às gestantes e parturientes. A maior inadequação encontrada foi relativa à solicitação desnecessária de teste rápido anti-HIV. Embora esta inadequação não gere um aumento direto do risco da transmissão, pode contribuir para a demora do resultado às mulheres que realmente necessitam do teste, postergando assim as ações de controle da transmissão vertical. Deve ser ressaltado também o prejuízo ao aleitamento materno ao nascimento, que é protelado se o resultado do teste rápido não está disponível.14

Faz-se necessário, portanto, fortalecer as políticas públicas para a adequação das ações de controle da transmissão vertical do HIV. A operacionalização da assistência primária deve ser qualificada, com ênfase na captação precoce das gestantes para o pré-natal e na ampliação do uso do teste rápido. A infraestrutura dos serviços laboratoriais deve ser aprimorada, com a disponibilização de exames e resultados em tempo oportuno. Por fim, os profissionais de saúde envolvidos na assistência a gestantes e parturientes necessitam ser capacitados, inclusive em serviço, nas ações de aconselhamento e manejo clínico.

\section{Referências}

1. Kellerman SE, Ahmed S, Feeley-Summerl T, Jay J, Kim M, Phelps BR, Sugandhi N, Schouten E, Tolle M,Tsiouris F Child Survival Working Group of the Interagency Task Team on the Prevention and Treatment of HIV infection in Pregnant Women, Mothers and Children. Beyond prevention of mother-to-child transmission: keeping HIV-exposed and HIV-positive children healthy and alive. AIDS. 2013; 27 (Suppl 2): S225-33.

2. Brasil. Ministério da Saúde. Transmissão vertical do HIV sífilis: estratégias para redução e eliminação. Brasília, DF; 2014

3. Barreto ML, Teixeira MG, Bastos FI, Ximenes RA, Barata $\mathrm{RB}$, Rodrigues LC. Successes and failures in the control of infectious diseases in Brazil: social and environmental context, policies, interventions, and research needs. Lancet. 2011; 377: 1877-89.

4. Brasil. Ministério da Saúde. Secretaria de Vigilância em Saúde. Programa Nacional de DST e AIDS. Boletim Epidemiológico - AIDS e DST- até a semana epidemiológica $27^{\mathrm{a}}$ a $52^{\mathrm{a}}$ semanas epidemiológicas - julho a dezembro de $2013 ; 1^{\mathrm{a}}$ a $26^{\mathrm{a}}$ semanas epidemiológicas janeiro a junho de 2014. Brasília, DF; 2014

5. Centro de Referência e Treinamento em DST/AIDS - São Paulo. Eliminação da transmissão vertical do HIV e da sífilis no Estado de São Paulo. Rev Saúde Pública. 2011; 45 (4): $812-5$.

6. Araújo ESP, Friedman RK, Camacho LAB, Derrico M, Moreira RI, Calvet GA, Santini de Oliveira M, Gonçalves Veloso V, Pilotto JH, Grinsztejn B. Cascade of access to interventions to prevent HIV mother to child transmission in the metropolitan area of Rio de Janeiro, Brazil. Braz J Infect Dis. 2014; 18 (3): 252-60.

7. Domingues RMSM, Szwarcwald CL, Souza Jr PRB, Leal MC. Prenatal testing and prevalence of HIV infection during pregnancy: data from the "Birth in Brazil" study, a national hospital-based study. BMC Infect Dis. 2015; 15:100.

8. Brasil. Ministério da Saúde. Manual técnico pré-natal e puerpério: atenção qualificada e humanizada. Brasília: Ministério da Saúde, 2006. 
9. Brasil. Ministério da Saúde. Secretaria de Vigilância em Saúde. Recomendações para profilaxia da transmissão vertical do HIV e terapia anti-retroviral em gestantes. Série: Manuais n 46. Brasília, DF; 2007.

10. Brasil. Ministério da Saúde. Protocolo para a prevenção de transmissão vertical de HIV e sífilis - manual de bolso. Programa Nacional de DST e Aids. Brasília, DF; 2007.

11. Brasil. Ministério da Saúde. Recomendações para profilaxia da transmissão vertical do HIV e terapia anti-retroviral em gestantes - manual de bolso. Programa Nacional de DST e Aids. Brasília, DF; 2010.

12. Ferreira-Junior OC, Ferreira C, Riedel M, Widolin MR, Barbosa-Júnior A; HIV Rapid Test Study Group. Evaluation of rapid tests for anti-HIV detection in Brazil. AIDS. 2005; 19 (Suppl. 4): S70-5.

13. Domingues RMSM, Hartz ZMA, Leal MC. Avaliação das ações de controle da sífilis e do HIV na assistência pré-natal da rede pública do município do Rio de Janeiro, Brasil. Rev Bras Saúde Matern Infant. 2012; 12(3): 269-80.

14. Oliveira MIC, Silva KS, Gomes-Jr SC, Fonseca VM. Resultado do teste rápido anti-HIV após o parto: uma ameaça à amamentação ao nascimento. Rev Saúde Pública. 2010; 44 (1): 60-9

15. Brousselle A, Champagne F, Contandriopoulos A-P, Hartz Z (org.). Avaliação: conceitos e métodos. Rio de Janeiro Editora Fiocruz; 2011

16. Oliveira MIC, Hartz ZMA, Nascimento VC, Silva KS Avaliação da implantação da iniciativa hospital amigo da criança no Rio de Janeiro, Brasil. Rev Bras Saúde Matern Infant. 2012; 12 (3): 281-95.

17. Cochran WG. Sampling Techniques. Wiley Series. In Probability and Statistics. Ed: IE-WILEY; 1977.

18. McLaughlin JA, Jordan GB. Logic models: a tool for telling your program's performance story. Eval Prog Plan. 1999; 22: $65-72$.

19. Cerda R, Perez F, Domingues RMSM, Luz PM, Grinsztejn B, Veloso VG, Caffe S, Francke JA. Prenatal transmission of syphilis and human immunodeficiency virus in Brazil achieving regional targets for elimination. Open Forum Infect Dis. 2015; 2 (2): ofv073.

20. Souza Junior PRB, Szwarcwald CL, Barbosa Junior A, Carvalho MF, Castilho EA. Infecção pelo HIV durante a gestação: estudo sentinela parturiente, Brasil, 2002. Rev Saúde Pública. 2004; 38: 764-72.

Recebido em 15 de maio de 2015

Versão final apresentada em 24 de setembro de 2015

Aprovado em 01 de outubro de 2015
21. Rodrigues CS, Guimarães MDC, César CC. Missed opportunities for congenital syphilis and HIV perinatal transmission prevention. Rev Saúde Pública. 2008; 42 (5): 851-8.

22. Szwarcwald CL, Barbosa Júnior A, Souza Júnior PRB, Lemos KRV, Frias PG, Luhm KR, Holcman MM, Esteves MA. HIV testing during pregnancy: use of secondary data to estimate 2006 test coverage and prevalence in Brazil. Braz J Infect Dis. 2008; 12 (3): 167-72.

23. Santos EM, Reis AC, Westman S, Alves RG. Avaliação do grau de implantação do programa de controle da transmissão vertical do HIV em maternidades do "Projeto Nascer”. Epidemiol Serv Saúde. 2010; 19 (3): 257-69.

24. Veloso VG, Portela MC, Vasconcellos MT, Matzenbacher LA, Vasconcelos AL, Grinsztejn B, Bastos FI. HIV testing among pregnant women in Brazil: rates and predictors. Rev Saúde Pública. 2008; 42 (5): 859-67.

25. Lemos LMD, Gurgel RQ, Fabbro ALD. Prevalência da infecção por HIV em parturientes de maternidades vinculadas ao SUS. Rev Bras Ginecol Obstet. 2005; 27 (1): 32-6.

26. Santos NP, Castro BG, Grassi MFR. Aplicação do protocolo do "Projeto Nascer Maternidades" em uma maternidade de referência em Feira de Santana, Bahia, Brasil. Rev Bras Saúde Matern Infant. 2009; 9 (1): 69-76.

27. Ramos V de OX, Lacerda HR, Ximenes RA de A. Unawareness of HIV status in pregnancy, delay in testing and conflict between information on antenatal card and interview in Recife, Brazil. Int J STD AIDS. 2009; 20: $493-$ 8

28. Mendoza-Sassi RA, Cesar JA, Teixeira TP, Ravache C, Araújo GD, Silva TC. Diferenças no processo de atenção ao pré-natal entre unidades da Estratégia Saúde da Família e unidades tradicionais em um município da Região Sul do Brasil. Cad Saúde Pública. 2011; 27 (4): 787-96.

29. Morimura MCR, Mendes MDC, Souza AI, Alencar LCA. Frequência de testagem rápida para o HIV durante a admissão para o parto em puérperas no Instituto Materno Infantil Prof. Fernando Figueira, IMIP. Rev Bras Saúde Matern Infant. 2006; 6 (Supl. 1): 569-76.

30. Miranda AE, Filho ER, Trindade CR, Gouvêa GM, Costa DM, Oliveira TG, et al. Prevalência de sífilis e HIV utilizando testes rápidos em parturientes atendidas nas maternidades públicas de Vitória, Estado do Espírito Santo. Rev Soc Bras Med Trop. 2009; 42 (4): 386-91. 